\title{
Metformina: Uso clínico y actualización
}

\author{
Metformin: Clinical use and update \\ Irma Isabel de Vicente Aguilera, ${ }^{1}$ Miguel Osejo Betancourt, ${ }^{1}$ Luis Alfredo Rodríguez, ${ }^{2}$ \\ Scarlet Betzabel Rodas Gallardo, ${ }^{3}$ María Alejandra Ramos Guifarro, ${ }^{4}$ Dalia María Ávila Turcios. ${ }^{4}$ \\ ${ }^{1}$ Médico Especialista en Medicina Interna, Hospital Escuela (HE). \\ ${ }^{2}$ Médico General, Instituto Nacional del Diabético. \\ ${ }^{3}$ Estudiante de Sexto Año, Carrera de Medicina, Facultad de Ciencias Médicas, UNAH. \\ ${ }^{4}$ Médico Especialista en Medicina Interna y Endocrinología, HE; Tegucigalpa.
}

\begin{abstract}
RESUMEN. La metformina (biguanida), grupo de medicamentos que proceden de la guanidina, se ha utilizado desde época medieval para tratamiento de la diabetes. Esta revisión bibliográfica narrativa tiene el propósito de contribuir a mejorar su uso clínico. Se realizó búsqueda de artículos originales, revisiones sistemáticas y artículos de revisión en internet, período 2012-2018, o anterior si fuera relevante. La metformina actúa como un hipoglucemiante, reduce la producción hepática de glucosa inhibiendo la gluconeogénesis y la glucogenólisis, aumenta captación de glucosa a nivel muscular y disminuye absorción de glucosa a nivel gastrointestinal. Una vez intracelular, aumenta la glucólisis anaerobia, uno de sus principales efectos adversos. La metformina es un fármaco que genera incremento de sensibilidad a insulina, mayor control de la glucemia, mejoría del perfil lipídico y de la función vascular, es de bajo costo y es en la actualidad la primera opción en el tratamiento de la diabetes mellitus tipo 2.
\end{abstract}

Palabras clave: Diabetes mellitus; Diabetes mellitus, tipo 2; Metformina.

\section{INTRODUCCIÓN}

La metformina pertenece a la familia de las biguanidas, grupo de medicamentos que proceden de la guanidina que se derivan de la planta Galega offininalis, la cual se ha utilizado desde la época medieval para el tratamiento de la diabetes. ${ }^{1-3}$ Las biguanidas fueron sintetizadas desde 1920, pero su uso terapéutico no obtuvo tanta validez dado que coincidió con la implementación de la insulina, hasta que en 1957 se comenzó a utilizar la fenformina y buformina las cuales fueron retiradas de muchos países a comienzos de la década de los 70's por su asociación con la aparición de acidosis láctica y oficialmente en 1977 la administración de alimentos y medicamentos de Estados Unidos (FDA) ordeno que fuesen retirados del mercado por su asociación con la aparición de acidosis láctica. ${ }^{1-4}$

A finales de la década de 1950 se introdujo la metformina, utilizándose en grandes cantidades como tratamiento de elección de la diabetes mellitus tipo 2 (DM2). Actualmente es la única biguanida disponible ya que resultó más segura reduciendo los niveles de glucemia sin peligro de causar hipoglicemias y su asociación con la incidencia de acidosis láctica es muy baja. ${ }^{1-3}$ Con más de 60 años de uso, se ha convertido en el antidiabético oral recetado con más frecuencia en la mayoría de países ya que es el fármaco de elección en el esquema básico de tratamiento. Sin embargo, su utilidad como antidiabético no es la única demostrada. ${ }^{1,3}$

Esta revisión bibliográfica narrativa se realizó con el propósito de actualizar la información de la metformina y contribuir a mejorar su uso clínico. Se realizó una búsqueda de artículos originales, revisiones sistemáticas y artículos de revisión biblio-

\footnotetext{
Recibido 14-12-2018; Aceptado para publicación 7-3-2019

Dirigir correspondencia a: Dra. Irma Isabel de Vicente Aguilera

Correo electrónico: irmadevicente@yahoo.com

Conflictos de interés. Los autores declaramos no poseer conflictos de interés en relación a este artículo.

DOI: https://doi.org/10.5377/rmh.v87i1.11935
}

gráfica en internet. Se realizó la búsqueda para los años entre 2012 y 2018. En vista de identificar dos artículos relevantes anteriores, se incluyeron en la revisión como excepción.

\section{MECANISMO DE ACCIÓN DE LA METFORMINA}

El mecanismo exacto por el que la metformina funciona aún no se ha documentado; actúa como un antihiperglucemiante, pero no predispone a hipoglucemia ${ }^{2}$. Se conoce que reduce la producción hepática de glucosa por inhibición de la gluconeogénesis y de glucogenólisis, aumenta la captación de glucosa a nivel muscular y disminuye la absorción de glucosa a nivel del tracto gastrointestinal. En el torrente sanguíneo una vez dentro de la célula, aumenta la glucólisis anaerobia el cual es uno de sus principales efectos adversos. ${ }^{1}$ No hay indicios que la metformina se metabolice, su absorción principalmente ocurre en el intestino delgado a través de los receptores de monoaminas de la membrana plasmática las cuales se excretan sin modificación por el riñón mediante filtración y transporte activo de Receptores Orgánicos de Cationes - 2 (OCT-2) ${ }^{1}$. El paso al espacio intracelular se da por los Receptores Orgánicos de Cationes (OCT) que están divididos en cinco subtipos; en los que el OCT1 y OCT-3 son los principales implicados en su función antidiabética porque su localización principal es en los hepatocitos. Sin embargo, se conoce que su efecto ocurre por la inhibición en la cadena respiratoria de la mitocondria de manera específica en el complejo I, sin afectar a los demás complejos I, ${ }^{1,5}$ como se explica de manera esquemática en la Figura 1.

La metformina llega a los hepatocitos de manera activa mediante el transportador OCT-1, el cual es resultado de una inhibición de la cadena respiratoria mitocondrial, la gran actividad del transportador OCT-1 aumenta el índice de entrega de la metformina en el espacio intracelular por lo tanto aumenta su efecto. La metformina posee una carga positiva por lo cual se acumula en las células y en las mitocondrias debido a los poten- 


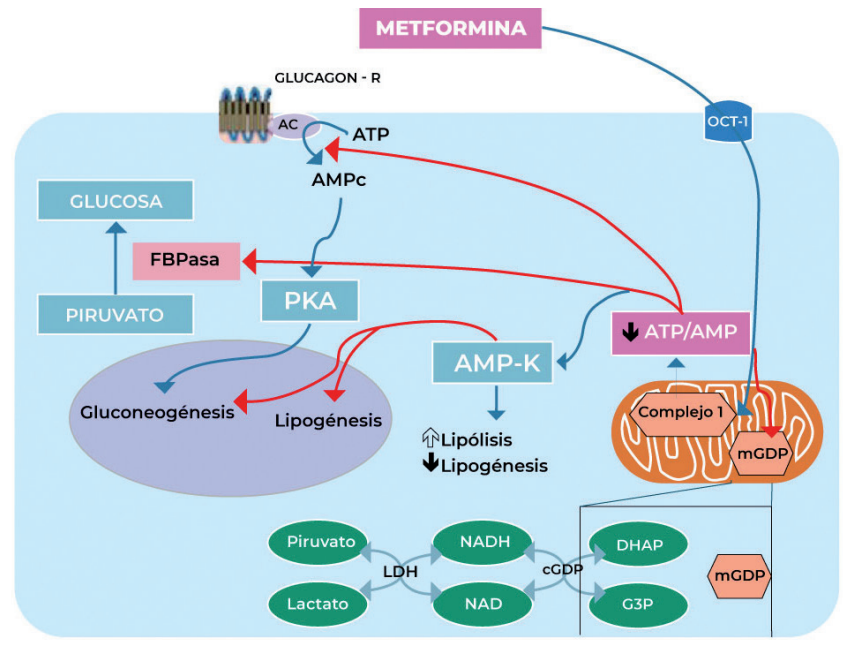

Figura 1. Esquema del efecto anti-hiperglicemiante de la metformina en el hepatocito. Adaptado de Morantes-Caballero J, Londoño-Zapata G, Rubio-Rivera M, Pinilla-Roa A. Metformina: más allá del control glucémico. Médicas UIS 2017; 30(1):57-71 (Referencia 6).

ciales de membrana por medio de la membrana plasmática y la membrana interna mitocondrial. Al interior de las células hepáticas, la metformina induce una inhibición leve de la acción postoxidativa modificando el estado de energía medida, mediante aumento de las proporciones citoplásmicas de ADP: ATP y AMP; estos cambios activan AMPK, la relación de ADP- ATP, reduce el índice de producción de glucosa, la activación de la proteína quinasa activada por AMP también llamada AMPK promueve una mayor acción de la insulina y reduce la tasa de gluconeogénesis y de la glucogenólisis. A niveles anormalmente altos de glucagón se genera una mayor producción de glucosa hepática, la metformina reduce la respuesta cíclica del AMP a la estimulación del glucagón en los hepatocitos, lo que ocasiona cambios en la proteína quinasa $\mathrm{A}$, inhibiendo la gluconeogénesis y así reduciendo la producción de glucosa hepática y la hipoglicemia basal. ${ }^{6,7}$ La razón de ATP/AMP disminuye porque aumenta el AMP; esto da como resultado que el déficit de ATP disminuye la glucólisis que es necesaria durante dicho proceso enzimático. ${ }^{6}$, ${ }^{7} \mathrm{AMPK}$ es una proteína quinasa que funciona como el regulador maestro del metabolismo, existen 12 posibles combinaciones de isoformas, aunque no está claro si existen diferencias funcionales entre ellas, pero si existen diferencias en la distribución tisular, por ejemplo: los heterotrimeros a1 están presentes en hígado y tejido adiposo, mientras que las a2 se encuentran en cerebro, musculo esquelético y musculo cardiaco. ${ }^{2}$

Para que AMPK pueda ser activada requiere de dos condiciones; incrementar la relación intracelular de AMPIATP y la fosforilación del "asa activadora" de la subunidad catalítica $\alpha$, una vez activada la AMPK es capaz de fosforilar una serie de proteínas, la mayoría de ellas con gran actividad enzimática. ${ }^{2}$ Los efectos de la activación de AMPK por la metformina tiene acción en múltiples tejidos y órganos, destacándose que en músculo esquelético aumenta la lipólisis y lipogénesis, a nivel cardiaco incrementa la captación de ácidos grasos, a nivel hepático reducen ácidos grasos y la síntesis de colesterol, incrementando la oxidación de lípidos y disminuyendo la gluconeogénesis, en tejido adiposo reduce la lipólisis y lipogénesis, en islotes pancreáticos específicamente en las células $\beta$ estimulan la secreción de insulina, por todos los efectos anteriormente mencionados en la activación de la AMPK actualmente se intenta obtener compuestos que puedan activar directa o indirectamente dicha enzima. ${ }^{1,2}$ En conclusión, genera pérdida de peso, incremento de sensibilidad a insulina, mayor control de la glucemia, mejoría del perfil lipídico y de la función vascular. Las vías catabólicas que aumentan la cantidad de ATP (ejemplo: consumo de glucosa y glucólisis) se activan por la enzima AMPK, mientras que las vías anabólicas consumidoras de ATP y otros procesos no esenciales (ejemplo: síntesis de proteínas, gluconeogénesis) se desactivan. ${ }^{1}$

\section{INDICACIONES DE LA METFORMINA}

\section{Como prevención de Diabetes}

Existe evidencia de que la administración de metformina disminuye la progresión de prediabetes a diabetes y, a pesar de ser menos efectiva como monoterapia que los cambios en el estilo de vida, el buen perfil de bioseguridad y el bajo costo hacen de este fármaco el de elección en el tratamiento conjunto. De hecho, el estudio Biguanidas y Prevención del Riesgo de Obesidad (BIGPRO) demostró que esta aproximación terapéutica modificó las anormalidades metabólicas asociadas con la resistencia a la insulina en individuos que no tenían diabetes, pero sí obesidad central, como: pérdida de peso, disminución de la glucosa e insulina en ayuno, del colesterol LDL, del activador tisular de plasminógeno 1 y de la proteína $\mathrm{C}$ reactiva. ${ }^{3}$

Asimismo, otros estudios, como el Programa para la Prevención de Diabetes (DPP) y el estudio Modificaciones en el Riesgo Cardiovascular en Sujetos Obesos y con Sobrepeso (CARMOS), han demostrado efectos deseables, por ejemplo, la metformina disminuye en aproximadamente $31 \%$ la aparición de diabetes en pacientes con una curva de tolerancia a la glucosa anormal y realizar cambios en el estilo de vida en conjunto con la metformina puede ser una alternativa de tratamiento de los factores de riesgo cardiovascular. Además, en los pacientes con síndrome metabólico se observó aumento del colesterol HDL, disminución de la glucosa de ayuno y del colesterol LDL. Por lo que la incidencia de diabetes en sujetos obesos y con sobrepeso disminuyó considerablemente, así como el perfil de riesgo cardiovascular en los pacientes con síndrome metabólico. ${ }^{3}$ Un estudio realizado por Pereira y Palay, en pacientes con obesidad exógena en la consulta externa de endocrinología del Hospital de Santiago de Cuba utilizando metformina junto a dieta, demostró que el tratamiento con metformina resultó ser más efectivo que la dieta sola, en la pérdida de peso de los pacientes con obesidad exógena, con lo cual se confirmó, entonces, la superioridad de dicha terapia, mejorando el perfil lipídico y la glicemia. ${ }^{8}$

\section{Diabetes Mellitus tipo 2}

Tanto el documento conjunto de las sociedades de diabetes estadounidense (ADA) y europea (EASD) sobre el tratamiento de la DM2 como la guía de la Federación Internacional de Diabetes (IDF) consideran metformina como el fármaco de primera elección en monoterapia, reservando los demás fár- 
macos orales y la insulina para el tratamiento combinado con metformina. ${ }^{9}$ Tradicionalmente se recomendaba empezar con metformina en pacientes con sobrepeso y con un secretagogo en los pacientes con normopeso. Sin embargo, ambas guías consideran que siempre es de elección metformina, ya que tiene claras ventajas sobre el resto de los fármacos orales puesto que mejora la sensibilidad a la insulina y no comporta aumento de peso ni hipoglucemias. ${ }^{9}$

La prevención de las complicaciones cardiovasculares es el resultado potencialmente más importante del tratamiento de la DM2. Las pruebas disponibles sugieren que estas metas son alcanzables con metformina, razón por la cual se recomienda como medicamento de primera elección en las guías de práctica clínica nacionales e internacionales actualizadas a 2018, así como en revisiones sistemáticas recientes sobre el tema. Otros fundamentos que ubican a la metformina como fármaco de primera elección en DM2 que requiere tratamiento con hipoglucemiantes, incluyen la larga experiencia de uso acumulada, su baja tendencia a producir hipoglucemia o ganancia de peso, la posibilidad de combinarla con fármacos de todas las otras clases de hipoglucemiantes, la comprobación del riesgo extremadamente bajo de acidosis láctica, la posibilidad de utilizarla aún con deterioro leve a moderado de la función renal, y el bajo costo del tratamiento. ${ }^{10}$

Esta posición de privilegio de la metformina viene avalada por numerosos ensayos clínicos y metaanálisis que demuestran que su uso está asociado con una disminución significativa en la mortalidad cardiovascular más allá del control glucémico, sugiriendo la implicación de diferentes mecanismos vásculoprotectores, y resaltando además su bajo riesgo de hipoglucemia. ${ }^{12}$ La dosis máxima de metformina habitualmente recomendada es de $2 \mathrm{~g} / \mathrm{dí}$. Dosis más altas se asocian con mayor frecuencia de efectos adversos gastrointestinales y con anemia causada por deficiencia de vitamina B12 sin lograr una reducción de la glucosa en ayunas y de la hemoglobina glucosilada ( $\mathrm{HbA} 1 \mathrm{c})$ significativamente diferentes. ${ }^{11}$

\section{Diabetes Mellitus tipo 1}

En el estudio REMOVAL se utilizó metformina en pacientes con diabetes de tipo $1 \mathrm{y}$ alto riesgo cardiovascular. El ensayo evaluó los efectos de metformina sobre el grosor de capa íntima media de la arteria carótida como criterio de valoración subrogado de ateroesclerosis, así como en parámetros metabólicos. Los resultados mostraron que en pacientes tratados con metformina no hubo mejoría en el grosor carotideo, ni en los niveles de $\mathrm{HbA}_{1 \mathrm{c}}$ o los requerimientos de insulina a tres años, aunque si hubo una tendencia a disminuir el peso y los niveles de colesterol LDL. ${ }^{12}$

\section{Uso en el embarazo}

En la diabetes gestacional por su buen perfil de seguridad es la primera línea de tratamiento después de la intervención nutricional, reduce el riesgo de hipoglicemia, aumento de peso versus otros tratamientos, no hay evidencia que provoque malformaciones congénitas o abortos. En seguimiento de los bebes nacidos de madre que la consumieron durante el embarazo no hubo diferencias en el desarrollo motor o lingüístico a los 2 años de nacido, efectos de esta a largo plazo aún no han sido definidos. ${ }^{13,14}$

\section{Síndrome de ovario poliquístico}

Es el desorden hormonal más común en la mujer en edad reproductiva, caracterizado por Oligo y / o anovulación, Signos clínicos y / o bioquímicos de hiperandrogenismo y Ovarios poliquísticos documentado por ultrasonido, provocando un mayor riesgo de infertilidad, dislipidemia, hiperplasia endometrial, metabolismo anormal de la glucosa. ${ }^{15,16}$

La hiperinsulinemia que muchas veces acompaña a esta patología aumenta la producción ovárica de andrógenos e inhibe la producción hepática de globulina fijadora de hormonas sexuales, aumentando aún más los niveles circulantes de testosterona libre. También, la insulina impide la ovulación, ya sea afectando directamente el desarrollo folicular o aumentando indirectamente los niveles de andrógenos intraováricos 0 alterando la secreción de gonadotropinas. La metformina actúa al inhibir la producción de glucosa hepática y aumenta la sensibilidad de los tejidos periféricos a la insulina; reduce los valores séricos de la misma y así disminuye los niveles de testosterona en aproximadamente 20 a $25 \%$ en mujeres con síndrome de ovario poliquístico. Sin embargo, sus efectos sobre el hirsutismo, irregularidad menstrual, lípidos no se han demostrado, si se encontró un beneficio en la disminución del índice de masa corporal y obesidad abdominal asociada con el estilo de vida. ${ }^{15-19}$

\section{Riesgo Cardiovascular}

Se considera que la metformina puede ser beneficiosa para la enfermedad cardiovascular pero no hay evidencia solidad que lo respalda. Este beneficio no parece estar relacionado con el control de la glicemia, pero aún no está dilucidado. ${ }^{20,21}$

\section{EFECTOS ADVERSOS}

Los efectos adversos más frecuentes con el consumo de metformina son alteraciones digestivas, principalmente: náuseas, vómitos, diarrea, disgeusia, dolor cólico y molestias abdominales. Estos síntomas podrían ser producto de la acumulación del fármaco en los enterocitos en el intestino delgado, generalmente son leves y transitorios, asociados a la dosis que ingiere el paciente. Alrededor de un tercio de pacientes los presentan al iniciar la terapia por lo que se recomienda iniciar a dosis bajas una o dos veces al día y aumentar cada semana hasta alcanzar la dosis efectiva tolerada o reducir la dosis en casos de intolerancia al fármaco. El consumo del medicamento con las comidas también se recomienda para ayudar a que los síntomas disminuyan. Alrededor de un $90 \%$ tolera el consumo crónico del medicamento y sólo el $5 \%$ de los pacientes no toleran siquiera las dosis más bajas. ${ }^{22,23}$ Uno de los efectos adversos más temidos con las terapias para el tratamiento de la diabetes es la hipoglicemia. La metformina se sitúa como primera elección en el tratamiento de la diabetes mellitus tipo 2 no sólo por los efectos favorables que se obtienen con su uso, sino también por su bajo riesgo de hipoglicemia al no aumentar las concentraciones de insulina. ${ }^{23,24}$ En estudios como el United Kingdom Prospective Diabetes Study (UKPDS) se registró una incidencia 
de 0,6 episodios al año, inferior que las incidencias de hipoglicemias en pacientes con otros esquemas de tratamiento. ${ }^{25}$

Se ha asociado el consumo de metformina a un incremento en el riesgo de desarrollar acidosis láctica, una reacción infrecuente pero peligrosa que postergó su introducción en el mercado de Estados Unidos y produjo el retiro de otras biguanidas como la butformina y fenformina. ${ }^{22-24}$ Aunque se calcula que el riesgo de acidosis láctica por metformina es 20 veces menos frecuente que con fenformina, se estima una incidencia de 3 a 10 por 100.000 personas por año y una mortalidad incluso cercana al $40 \% .{ }^{22,25} \mathrm{~A}$ pesar que diversos estudios clínicos no presentan casos de acidosis láctica por metformina y que la presencia de esta reacción es casi exclusiva de pacientes con riesgo alto independiente del tratamiento con metformina, no se descarta el hecho de que la metformina agregue un riesgo adicional de que un paciente predispuesto la desarrolle. ${ }^{23,24}$ Los pacientes con riesgo alto de presentarla son principalmente aquellos con edad superior a 65 años o enfermedades de base que tienen capacidad de producir hipoxia y desencadenar acidosis láctica como ser: insuficiencia renal crónica, insuficiencia cardiaca congestiva, evento cardiovascular, shock cardiogénico, enfermedad pulmonar severa, exceso de alcohol o sepsis, hepatopatía avanzada e historia de acidosis láctica previa, además de la administración de contrastes yodados. ${ }^{22,24,25}$ Mientras algunos mencionan que no está clara la relación entre la acumulación de metformina y la acidosis láctica. Este evento ocurre cuando las concentraciones séricas del fármaco llegan a niveles tóxicos $(>5.0 \mathrm{mg} / \mathrm{L})$ sobrepasando los niveles terapéuticos $(0.5-2.0 \mathrm{mg} / \mathrm{L})$, que en la mayoría de los casos es ocasionado por disminución de la filtración renal. ${ }^{22,25} \mathrm{Se}$ atribuye que la acidosis láctica es producto del aumento en la generación de lactato que resulta ya que se promueve el metabolismo anaeróbico por interferencia con la respiración mitocondrial. ${ }^{25}$

Otro de los efectos adversos que se pueden producir por consumo de metformina es la disminución progresiva de vitamina B12 que ocurre por malabsorción, aunque el mecanismo no está del todo claro. ${ }^{22,23,26}$ Se proponen algunas teorías para esto: una, el hecho de afectar la producción de ácido clorhídrico necesario para mantener el ambiente ácido que permite liberar la cobalamina de la matriz alimentaria; otra, afectar la producción de factor intrínseco secretado por las células parietales de la mucosa gástrica, que se une a la vitamina B12 liberada formando el complejo Cob-FI y la tercera teoría es la inhibición de la cubilina, un transportador dependiente de calcio de la membrana de los enterocitos necesaria para el transporte del complejo Cob-FI para ingresar a las células endoteliales. ${ }^{26} \mathrm{~A}$ pesar de que no se cuenta con información que demuestre que estas concentraciones bajas de vitamina B12 produzcan síntomas clínicos, se sugiere realizar pruebas periódicas en pacientes que estén en tratamiento con metformina, sobre todo si cursan con anemia o neuropatía periférica. Incluso el aporte profiláctico de vitamina B12 podría ser recomendado, en algunos casos hasta dosis relativamente altas de $1.000 \mu \mathrm{g} / \mathrm{día}^{22,23}$

\section{CONTRAINDICACIONES}

La recomendación general es que en todo paciente con diabetes mellitus tipo 2 inicie tratamiento con metformina desde el momento del diagnóstico o poco después, excepto en casos de contraindicación o de intolerancia. ${ }^{23,27}$ Aunque sólo el $5 \%$ de los pacientes no toleran incluso las dosis más bajas, se recomienda la interrupción en el consumo del medicamento en casos de diarrea, vómitos u otras causas de deshidratación que no permitan su tolerancia. ${ }^{22,25}$ También se recomienda la omisión del fármaco en los pacientes antes de la investigación con medios de contraste yodados aunque la FDA lo recomienda cuando la Tasa de Filtrado Glomerular (TFG) se encuentre entre 30 a $60 \mathrm{ml} / \mathrm{min}$ en caso de enfermedad hepática, alcoholismo o insuficiencia cardía$\mathrm{ca}$, o si se usa contraste arterial. ${ }^{25,26}$ Se debe valorar nuevamente la TFG 48 horas después del procedimiento y reiniciar el fármaco si la función renal se encuentra preservada. ${ }^{26}$

La metformina tiene una excreción renal del $90 \%,{ }^{27}$ por lo que en casos de insuficiencia renal se le ha visto relacionado con acidosis láctica por acumulación del medicamento. ${ }^{25}$ Inicialmente la FDA contraindicaba el uso del fármaco en base a valor de creatinina sérica, si se encontraba $\geq 1.5 \mathrm{mg} / \mathrm{dl}(\geq 114 \mu \mathrm{mol} / \mathrm{L})$ en varones $\mathrm{y} \geq 1.4 \mathrm{mg} / \mathrm{dl}(\geq 107 \mu \mathrm{mol} / \mathrm{L})$ en mujeres o mayores de 80 años con alteraciones en el aclaramiento de creatinina para evitar esta potencial complicación. ${ }^{22,23,27}$ Posteriormente, la FDA modificó la ficha técnica del fármaco ahora en base a la TFG según el Modification of Diet in Renal Disease (MDRD) y no en base a valores solos de creatinina sérica, recomendando su uso cuando la TFG $>60 \mathrm{ml} / \mathrm{min}$, aunque estudios recientes establecen que su uso es seguro hasta $30 \mathrm{~m} / \mathrm{min}$ ya que se mantienen dosis terapéuticas del fármaco en plasma. ${ }^{22,23,27}$ En los pacientes que la TFG baja hasta $50045 \mathrm{ml} / \mathrm{min}$ se debe hacer un análisis personal del paciente evaluando riesgo beneficio, continuándolo con precaución hasta una dosis diaria máxima de $1,000 \mathrm{mg}$ y omitirla cuando esta descienda a $30 \mathrm{ml} / \mathrm{min}^{22,23}$ Aunque diversos estudios establecen que el riesgo de desarrollar acidosis láctica es extremadamente bajo y no diferente al riesgo de los pacientes con otros hipoglicemiantes orales, ${ }^{22}$ recomiendan que con TGF $<45 \mathrm{ml} / \mathrm{min}$ se suspenda el fármaco si además presenta factores de riesgo para acidosis láctica, como los mencionados con anterioridad. ${ }^{27}$ Otra consideración a tener en cuenta es que los pacientes con bypass gástrico tienen un aumento en la biodisponibilidad del $50 \%$ del fármaco, por lo que la dosis podría ajustarse en grados más leves de enfermedad renal. ${ }^{25}$ Además se encuentra contraindicada en la insuficiencia hepática y el alcoholismo. ${ }^{23}$

\section{CONCLUSIÓN}

La metformina actúa como un antihiperglucemiante, reduce la producción hepática de glucosa inhibiendo la gluconeogénesis y la glucogenólisis, aumenta captación de glucosa a nivel muscular y disminuye absorción de glucosa a nivel gastrointestinal. Todos estos mecanismos permiten utilizarla en diferentes escenarios clínicos en los que está involucrada sobretodo la resistencia a la insulina. Su bajo costo le asegura el primer lugar en el tratamiento del paciente con Diabetes tipo 2 pero también en patologías que le preceden. La metformina es un fármaco que genera incremento de sensibilidad a insulina, mayor control de la glucemia, mejoría del perfil lipídico y de la función vascular. 


\section{CONTRIBUCION DE LOS AUTORES}

Todos los autores contribuyeron en igual medida para el análisis y redacción de la información presentada en esta publi- cación, así como en la revisión y aprobación de la versión final presentada.

\section{REFERENCIAS}

1. Arocha Rodulfo J, Navas Blanco T, Aure G, Palacios A. Metformina, el fármaco paradigma del siglo XXI. Med Interna [Internet]. 2017 [consultado 18 marzo de 2018]; 33(1):4-18. Disponible en: http://www.svmi.web.ve/ojs/index.php/medint/article/view/409

2. Gonzales-Mujica F. Drogas antidiabéticas diferentes de la insulina: mecanismos de acción. VITAE, Academia Biomédica Digital. [Internet]2016. [consultado 10 de junio de 2018];66:1-22. Disponible en: http://vitae.ucv.vel index pdf.php?module=articulo\&n=5316

3. Castro-Martínez MG, Castillo-Anaya V, Ochoa Aguilar A, Godínez-Gutiérrez SA. La metformina y sus aplicaciones actuales en la clínica. Med Int Méx [Internet]. 2014[consultado 10 de junio de 2018];30(5):562-574. Disponible en: https://biblat.unam.mx/es/revista/medicina-interna-de-mexico/articulo/lametformina-y-sus-aplicaciones-actuales-en-la-clinica

4. Bailey C. Metformin: historical overview. Diabetología [Internet]. 2017 [consultado 10 Junio de 2018];60(9):1566-1576. Disponible en: https://www.ncbi. nlm.nih.gov/pubmed/28776081

5. Rena G, Pearson ER, Sakamoto K. Molecular mechanism of action of Metformin: Old or New Insights. Diabetología [Internet]. 2013 [consultado 10 de junio 2018];56(9):1898-1906. Disponible en: https://www.ncbi.nlm.nih.gov/ pubmed/23835523

6. Morantes-Caballero JA, Londoño-Zapata GA, Rubio-Rivera M, PinillaRoa AE. Metformina: más allá del control glucémico. Médicas UIS [Internet]. 2017. [consultado 10 de junio de 2018]; 30(1):57-71. Disponible en: $\quad$ http://www.scielo.org.co/scielo.php?script=sci_abstract\&pid=S012103192017000100057\&lng=es\&nrm=iso

7. Rena G, Hardie D, Pearson E. The mechanisms of action of metformin. Diabetología [Internet] 2017. [consultado 10 de junio de 2018] ;60(9):15771585. Disponible en: https://www.ncbi.nlm.nih.gov/pubmed/28776086

8. Pereira-Despaigne CO, Palay-Despaigne MS. Eficacia de la metformina en pacientes con obesidad exógena. MEDISAN [Internet]. 2015 [consultado 16 de abril de 2018];19(2):215. Disponible en: http://scielo.sld.cu/scielo. php?script=sci_arttext\&pid=S1029-30192015000200010

9. Inzucchi SE, Bergenstal M, Buse JB, Diamant M, Ferrannini E, Nauck M, et al. Management of hyperglycaemia in type 2 diabetes: a patient-centered approach. Position statement of the American Diabetes Association (ADA) and the European Association for the Study of Diabetes (EASD). Diabetología [Internet]. 2012 [consultado 16 de abril de 2018] ; 55(6):1577-1596. Disponible en: https://link.springer.com/article/10.1007/s00125-012-2534O\#citeas

10. Fundación FEMEBA. Metformina en el tratamiento de la diabetes mellitus tipo 2. Foliadoc [Internet]. 2016[consultado el 16 de abril de 2018]. Supl. espec. Disponible en: http://www.fundacionfemeba.org.ar/farmacologia/foliadoc-noviembre-2016-metformina-en-el-tratamiento-de-la-diabetes-mellitustipo-2

11. Llinas-Castro R, Alvis-Estrada L, Castillo-Avila I. Evaluación de la prescripción de metformina en pacientes diabéticos tipo 2 de una institución de Atención Primaria en Salud en Cartagena de Indias, Colombia. Rev Clín Med Fam [Internet]. 2017 [consultado 16 de abril de 2018];10(1):12-17. Disponible en: http://scielo.isciii.es/scielo.php?script=sci_abstract\&pid=\$1699695X2017000100003

12. Gotes Palazuelos J. Uso de agentes hipoglucemiantes en diabetes de tipo 1 ¿Qué hay de nuevo?. Medscape [Internet]. 2017 [consultado 16 de abril de 2018]. Disponible en: https://espanol.medscape.com/verarticulo/5901903

13. Rowan J, Hague W, Gao W, Battin M, Moore P. Metformin versus insulin for

the Treatment of gestational diabetes. N Engl J Med [Internet]. 2008 [consultado 23 de enero de 2018];358:2003-2015 . Disponible en: http://www.nejm. org/doi/full/10.1056/NEJMoa0707193

14. Lindsay RS, Loeken MR. Metformin use in pregnancy: promises and uncertainties. Diabetología. 2017;60(9):1612-1619.

15. Williams T, Mortada R, Porter S. Diagnosis and Treatment of Polycystic Ovary Syndrome. Am Fam Physicianr [Internet]. 2016 [consultado 23 de enero de 2018];94(2):106-113. Disponible en: https:/www.aafp.org/ afp/2016/0715/p106.pdf

16. McCartney CR, Marshall JC. Polycystic Ovary Syndrome. N Engl J Med [Internet]. 2016 [consultado 23 de enero de 2018]; 375:54-64. Disponible en: https://www.neim.org/doi/full/10.1056/NEJMcp1514916

17. Sam S, Ehrmann D. Metformin therapy for the reproductive and metabolic consequences of polycystic ovary syndrome. Diabetología. 2017; 60(9):1656-1661.

18. Johnson NP. Metformin use in women with polycystic ovary syndrome. Ann Transl Med [Internet]. 2014 [consultado 23 de mayo de 2018];2(6):56. Disponible en: https://www.ncbi.nlm.nih.gov/pmc/articles/PMC4200666/

19. Naderpoor N, Shorakae S, de Courten B, Misso M, Moran L, Teede H. Metformin and lifestyle modification in polycystic ovary syndrome: systematic review and meta-analysis. Human Reproduction Update, [Internet]. 2015 [consultado 23 de enero de 2018];21(5):560-574. Disponible en: https://doi. org/10.1093/humupd/dmv025

20. Lexis $\mathrm{CPH}$, van der Horst ICC. Metformin for cardiovascular disease: promise still unproven. Lancet Diabetes Endocrinol [Internet]. 2014[consultado 23 de enero de 2018];2(2):94-5.

21. Griffin SJ, Leaver JK, Irving GJ. Impact of metformin on cardiovascular disease: a meta-analysis of randomised trials among people with type 2 diabetes. Diabetologia. 2017; 60(9):1620-1629.

22. Sánchez Rangel E, Inzucchi SE. Metformin: clinical use in type 2 diabetes. Diabetología [Internet]. 2017[Consultado 14 de marzo de 2018];60:15861593. Disponible en: https://doi.org/10.1007/s00125-017-4336-x.

23. Melmed S, Polonsky KS, Larsen PR, Kronenberg HM. Williams. Tratado de endocrinología. 13a ed. Barcelona: Elsevier; 2017.

24. Carral San Laureano F, Ayala Ortega MC. Metformina y acidosis láctica: ¿asociación infrecuente o causalidad? Av Diabetol [Internet]. 2010 [Consultado 20 de febrero de 2018]; 26:273-5. Disponible en: https://www.sciencedirect.com/science/article/pii/S113432301064015X

25. Zanchi A, Lehmann R, Philippe J. Antidiabetic drugs and kidney disease. Recommendations of the Swiss Society for Endocrinology and Diabetology. Swiss Med Wkly [Internet]. 2012 [Consultado el 30 de enero de 2018];142:13629. Disponible en: https://smw.ch/article/doi/smw.2012.13629/

26. Sánchez H, Masferrer D, Lera L, Arancibia E, Ángel B, Albala C. Déficit de vitamina B12 asociado con altas dosis de metformina en adultos mayores diabéticos. Nutr Hosp [Internet]. 2014[consultado 30 de enero de 2018];29(6):1394-1400. Disponible en: http://scielo.isciii.es/scielo. php?script=sci_arttext\&pid=S0212-16112014000600024.

27. Gómez Huelgas R, Martínez Castelao A, Artola S, Górrize JL, Górriz JL, Menéndez E. Documento de Consenso sobre el tratamiento de la diabetes tipo 2 en el paciente con enfermedad renal crónica\#. Nefrología [Internet]. 2014 [Consultado 28 de enero de 2018]; 34(1): 34-45. Disponible en: https:/l revistanefrologia.com/es-documento-consenso-sobre-el-tratamiento-diabetes-tipo-2-el-paciente-articulo-X0211699514053673

ABSTRACT. Metformin belongs to the family of biguanides, a group of medicines that come from guanidine, which has been used since medieval times for the treatment of diabetes. This review paper has the objective to contribute to extend its clinical use. We searched on line for original and review articles and systematic reviews from 2012-2018, or before if relevant. Metformin acts as an antihyperglycemic agent, reducing hepatic glucose production by inhibiting gluconeogenesis and glycogenolysis, increasing glucose uptake at the muscle level and also decreasing glucose uptake at the level of the gastrointestinal tract. Intracellularly it increases anaerobic glycolysis one of its main adverse effects. Metformin is a drug that generates increased sensitivity to insulin, greater glycemic control, improved lipid profile and vascular function, it is low cost and currently the first option for the treatment of diabetes mellitus type 2.

Keywords: Diabetes mellitus; Diabetes mellitus, Type 2; Metformin. 